\title{
Evidence of interatomic Coulombic decay in ArKr after Ar 2p Auger decay
}

\author{
Y Morishita $^{1}$, N Saito ${ }^{1}$, I H Suzuki ${ }^{1,2}$, H Fukuzawa ${ }^{3}$, X-J Liu ${ }^{3}$, K Sakai ${ }^{3}$, \\ G Prümper ${ }^{3}, \mathrm{~K} \mathrm{Ueda}^{3}, \mathrm{H}_{\text {Iwayama }}{ }^{4}, \mathrm{~K}_{\mathrm{Nagaya}}{ }^{4}, \mathrm{M} \mathrm{Yao}^{4}, \mathrm{~K} \mathrm{Kreidi}^{5}$, \\ M Schöffler $^{6}$, T Jahnke ${ }^{6}$, S Schössler ${ }^{6}$, R Dörner ${ }^{6}$, T Weber ${ }^{7}$, J Harries $^{8}$ \\ and Y Tamenori ${ }^{8}$
}

${ }^{1}$ National Institute of Advanced Industrial Science and Technology (AIST), NMIJ,

Tsukuba 305-8568, Japan

${ }^{2}$ Photon Factory, Institute of Materials Structure Science, Tsukuba 305-0801, Japan

${ }^{3}$ Institute of Multidisciplinary Research for Advanced Materials, Tohoku University,

Sendai 980-8577, Japan

${ }^{4}$ Department of Physics, Kyoto University, 606-8502 Kyoto, Japan

${ }^{5}$ DESY, Notkestrasse 85, 22607 Hamburg, Germany

${ }^{6}$ Goethe-Universität Frankfurt am Main, Max-von-Laue-Str 1, D-60438 Frankfurt, Germany

${ }^{7}$ Lawrence Berkeley National Lab, Berkeley, CA 94720, USA

${ }^{8}$ Japan Synchrotron Radiation Research Institute, Sayo, Hyogo 679-5198, Japan

E-mail: norio.saito@aist.go.jp, ueda@tagen.tohoku.ac.jp

Received 5 October 2007, in final form 20 November 2007

Published 8 January 2008

Online at stacks.iop.org/JPhysB/41/025101

\begin{abstract}
We have identified interatomic Coulombic decay (ICD) processes in the ArKr dimer following Ar 2p Auger decay, using momentum-resolved electron-ion-ion coincidence spectroscopy and simultaneously determining the kinetic energy of the ICD electron and the KER between $\mathrm{Ar}^{2+}$ and $\mathrm{Kr}^{+}$. We find that the spin-conserved ICD processes in which $\mathrm{Ar}^{2+}\left(3 \mathrm{p}^{-3} 3 \mathrm{~d}\right){ }^{1} \mathrm{P}$ and ${ }^{3} \mathrm{P}$ decay to $\mathrm{Ar}^{2+}\left(3 \mathrm{p}^{-2}\right){ }^{1} \mathrm{D}$ and ${ }^{3} \mathrm{P}$, respectively, ionizing the $\mathrm{Kr}$ atom, are significantly stronger than the spin-flip ICD processes in which $\operatorname{Ar}^{2+}\left(3 \mathrm{p}^{-3} 3 \mathrm{~d}\right){ }^{1} \mathrm{P}$ and ${ }^{3} \mathrm{P}$ decay to $\operatorname{Ar}^{2+}\left(3 \mathrm{p}^{-2}\right)^{3} \mathrm{P}$ and ${ }^{1} \mathrm{D}$, respectively.
\end{abstract}

(Some figures in this article are in colour only in the electronic version)

\section{Introduction}

About a decade ago, Cederbaum et al [1] proposed a new mechanism of electronic decay where the environment plays a role. For isolated atoms or molecules with an innervalence vacancy Auger decay is often energetically forbidden, but interatomic or intermolecular Coulombic decay (ICD) may occur when another species is in close proximity. Marburger et al [2] first observed the ICD process in $2 \mathrm{~s}$ ionized Ne clusters and later Jahnke et al [3] reported clear experimental evidence for ICD in $2 \mathrm{~s}$ ionized Ne dimers by identifying the process unambiguously using cold-target recoil ion momentum spectroscopy (COLTRIMS) [4, 5]. ICD is relevant to numerous physical, chemical and biological phenomena involving charge and energy transfers from atoms and molecules to their environment, and is thus of significant current interest. Here, we refer to only a small subset of the recent theoretical [6-9] and experimental [10-14] works on ICD.

ICD has also been predicted to take place following Auger decay, as a second step process [15]. Recently, Morishita et al [16] investigated ICD in Ar dimers after $2 p$ Auger decay using momentum-resolved electron-ion-ion coincidence spectroscopy (equivalent to COLTRIMS). To our knowledge, however, there has been no experimental report on ICD following Auger decay in hetero-dimers, although these seem to be the clearest prototype system for the investigation of ICD where one atom plays the role of the environment to the other. In the present work, we have extended this observation to hetero-dimers. Namely, we have investigated ICD in ArKr from the states populated by the Auger decay of the Ar $2 p$ inner-shell hole state. In the experiment, each slow electron, either the $\operatorname{Ar} 2 p$ photoelectron or the ICD electron, is recorded in coincidence with $\mathrm{Ar}^{2+}$ and $\mathrm{Kr}^{+}$, and the correlation between 
the kinetic energy of the ICD electron and the kinetic energy release (KER) of the two ions is obtained.

\section{Experiment}

The experiment was carried out at beamline 27SU [17] of SPring-8, using the 26 single-bunches $+2 / 29$ filling mode, which provides a single-bunch separation of 165.2 ns. Radiation polarized linearly in the vertical direction from the figure- 8 undulator [18] was monochromatized by the Hettrick-type high-resolution monochromator [19]: the photon bandwidth was set to $\sim 30 \mathrm{meV}$ at a photon energy of $262.54 \mathrm{eV}$, which is $13.9 \mathrm{eV}$ and $11.8 \mathrm{eV}$ above the atomic $\mathrm{Ar}$ $2 \mathrm{p}^{-12} \mathrm{P}_{3 / 2}$ and ${ }^{2} \mathrm{P}_{1 / 2}$ ionization thresholds.

The $\mathrm{Ar}-\mathrm{Kr}$ hetero-dimers were produced by expanding a mixture of argon and krypton gases at a flow-rate ratio of $6: 1$ and a total stagnation pressure of $3.3 \mathrm{~atm}$ at room temperature through a pinhole of $50 \mu \mathrm{m}$ diameter and $0.25 \mathrm{~mm}$ thickness. Under these conditions the cluster beam contains a mixture of $\mathrm{Ar}$ and $\mathrm{Kr}$ monomers, $\mathrm{Ar}$ and $\mathrm{Kr}$ dimers, $\mathrm{Ar}-\mathrm{Kr}$ heterodimers as well as larger clusters. The cluster beam is directed vertically and crosses the monochromatized radiation.

The momentum-resolved electron-ion-ion coincidence spectroscopy technique is based on recording the electron and ion times-of-flight (TOFs) with multi-hit two-dimensional position sensitive detectors [20-23]. Knowledge of the position and arrival time on the particle detectors, $(x, y, t)$, allows us to extract information on the linear momentum $\left(p_{x}, p_{y}, p_{z}\right)$ for each particle. The two TOF spectrometers are placed face to face, and the TOF spectrometer axis is horizontal and perpendicular to both the photon beam and the molecular beam. The TOF spectrometer for the electron is equipped with a hexagonal multi-hit position-sensitive delay-line detector of effective diameter of $120 \mathrm{~mm}$, while that for the ion is of effective diameter $80 \mathrm{~mm}$ [24]. A static extraction field and a uniform magnetic field are applied to the spectrometers so that all the electrons and ions ejected to the whole $4 \pi$ sr solid angle were collected by the detectors.

\section{Results and discussion}

Figure 1 shows ion-ion coincidence spectra. The $x$ and $y$ coordinates correspond to the TOFs of the first and the second ions of the coincidence pair. The spectrum in the left panel is illustrated using the raw data. The procedure to select only the events $(\mathrm{Ar}-\mathrm{Kr})^{3+} \rightarrow \mathrm{Ar}^{2+}-\mathrm{Kr}^{+}$from the raw data is to impose in off-line analysis an acceptance window on the sum of the momentum of the two ions $\mathrm{Ar}^{2+}$ and $\mathrm{Kr}^{+}$, using the momentum conservation law. This selection provides the spectrum in the right panel of figure 1 . $\mathrm{Ar}^{2+}$ and $\mathrm{Kr}^{+}$ions with zero momentum are located at TOFs of 3.27 and $6.70 \mu$ s, respectively. One can see clearly the lines corresponding to fragmentation into $\mathrm{Ar}^{2+}$ $\mathrm{Kr}^{+}$. It is worth noting that this selection rejects not only false coincidences coming from the monomer but also true ion-ion coincidences resulting from the explosion of larger clusters. In the present measurement, the counting rate of the $\mathrm{Ar}^{2+}-\mathrm{Kr}^{+}$ coincidence thus selected relative to the total ion counting rate is $0.05 \%$.

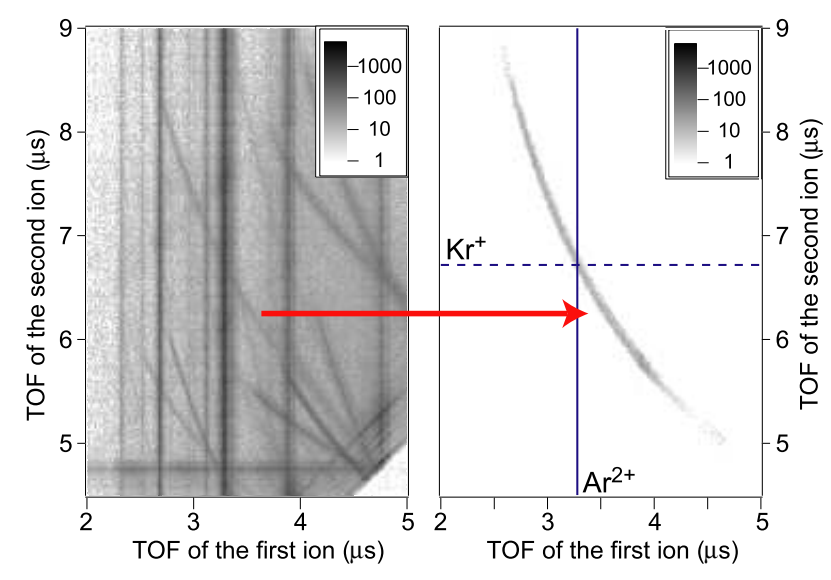

Figure 1. Ion-ion coincidence TOF spectra. The spectrum in the left panel is illustrated using the raw data, without any selections. The spectrum in the right panel is produced using the data selected from the raw data using the condition of the momentum conservation between the $\mathrm{Ar}^{2+}$ and $\mathrm{Kr}^{+}$ions. The line shows the time-of-flight for zero-momentum $\mathrm{Ar}^{2+}$ ions, the dotted line for zero-momentum $\mathrm{Kr}^{+}$ions.
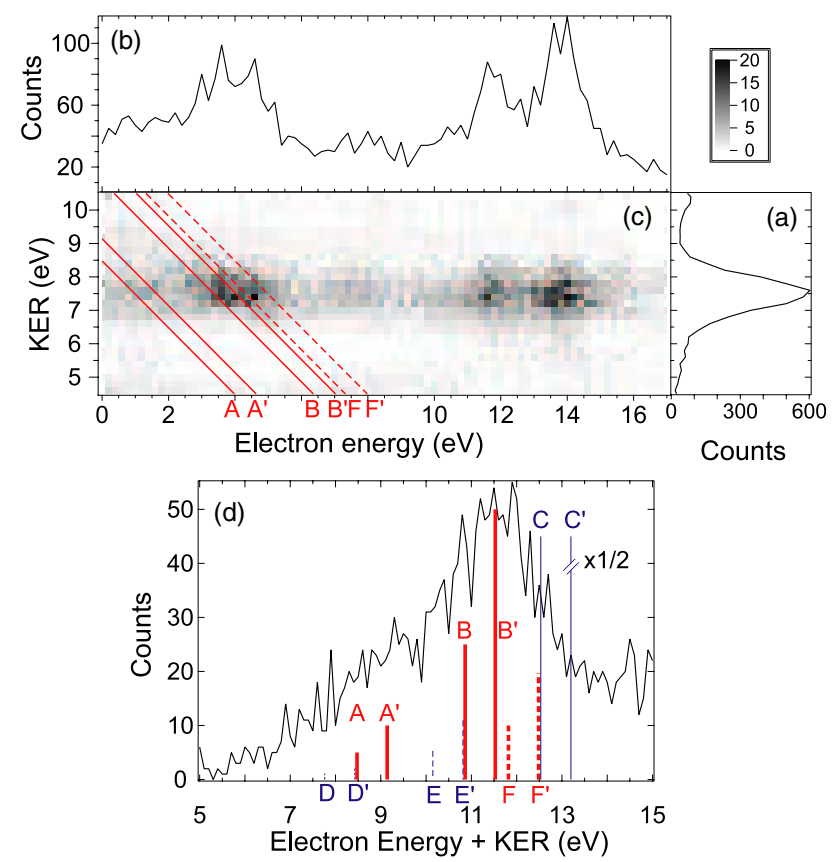

Figure 2. (a) Kinetic energy release (KER) distribution in $(\mathrm{ArKr})^{3+} \rightarrow \mathrm{Ar}^{2+}-\mathrm{Kr}^{+}$recorded in coincidence. (b) Energy distribution of the electron ejected from $\mathrm{ArKr}$ coincident with the $\mathrm{Ar}^{2+}$ and $\mathrm{Kr}^{+}$ions. (c) Relationship between the electron energy and the KER. (d) The sum of the electron energy and the KER. The solid lines correspond to the ${ }^{1} \mathrm{P}$ Auger final states (labels of A-C), and the dotted lines the ${ }^{3} \mathrm{P}$ states (labels of D-F). The thick lines $\mathrm{A}, \mathrm{B}$, and $\mathrm{F}$, (also shown in (c)), correspond to spin-conserved transitions, and the thin lines C, D, and E correspond to spin-flip transitions.

Figure 2(a) shows the distribution of the KER in $(\mathrm{ArKr})^{3+}$ fragmentation into the $\mathrm{Ar}^{2+}$ and $\mathrm{Kr}^{+}$ions. The peak energy of $7.47 \mathrm{eV}$ for the KER distribution corresponds to an internuclear distance of $3.85 \AA$, if one assumes a pure Coulomb explosion and neglects nuclear dynamics. This value is very close to 
the bond length of neutral ArKr, $3.88 \AA$ [25], suggesting that Auger decay followed by ICD is faster than the nuclear motion in both the core-ionized state and the Auger final dicationic states of ArKr.

Figure 2(b) shows the energy distribution of electrons coincident with fragmentation into $\mathrm{Ar}^{2+}-\mathrm{Kr}^{+}$. The two peaks at 11.8 and $13.9 \mathrm{eV}$ correspond to photoelectrons. A further, broader peak appears at $\sim 4 \mathrm{eV}$. This peak corresponds to the ICD electrons as will be discussed below.

Our coincidence measurement for one electron and two ions provides the electron kinetic energy together with the KER between the two ions for each event. The relationship of the electron energy and the KER in the fragmentation into $\mathrm{Ar}^{2+}-\mathrm{Kr}^{+}$is shown in figure 2(c). The two islands on the righthand side are attributed to photoelectrons as described above. The island on the left-hand side corresponds to ICD emission, as discussed below.

In order to make a clear discussion of the ICD processes, the distribution of the sum of the electron kinetic energy and the KER is plotted in figure 2(d). The region shown corresponds to the left-hand side of figure 2(c), and the vertical lines correspond to the energy sums for the ICD transitions, as discussed below.

The energy resolution of our spectrometer depends on the electron and ion energies, and is estimated as follows. The electron kinetic energy resolution is estimated to be $0.4 \mathrm{eV}$ at $4 \mathrm{eV}$ (ICD electrons) and $1.1 \mathrm{eV}$ at $14 \mathrm{eV}$ (photoelectrons). These estimates are derived from the time and position resolutions of the position-sensitive detector, and taking into account that the multiplet components of $\operatorname{Ar}^{2+}\left(2 \mathrm{p}^{-2}\right)$ are unresolved. The measured full-width at half-maximum of the photoelectron peak at $14 \mathrm{eV}$ is about $1.2 \mathrm{eV}$, indicating that our estimate is reasonable. The resolution of the ion KER is estimated to be $0.3 \mathrm{eV}$ at a KER of $7.5 \mathrm{eV}$, estimated from the time and position resolutions of the detector and considering that the $\mathrm{Kr}$ isotopes are not resolved. The overall energy resolution for the energy sum in figure $2(\mathrm{~d})$ is thus estimated to be $0.5 \mathrm{eV}$.

Figure 3 shows a schematic energy level diagram relevant to the ICD after Auger decay. In the independent particle (single-configuration) approximation, the two-vacancy Auger final states of atomic $\mathrm{Ar}^{2+}$ are $3 \mathrm{p}^{-2}, 3 \mathrm{~s}^{-1} 3 \mathrm{p}^{-1}$, and $3 \mathrm{~s}^{-2}$. In the case of atomic $\mathrm{Ar}^{2+}$, however, the independent particle approximation completely breaks down. For example, the dicationic states at 61.25 and $70.65 \mathrm{eV}$ above the neutral atomic ground state are usually assigned to $3 \mathrm{~s}^{-1} 3 \mathrm{p}^{-1}{ }^{1} \mathrm{P}$ and $3 \mathrm{p}^{-3} 3 \mathrm{~d}{ }^{1} \mathrm{P}$, respectively $[26,27]$. However, in reality, both configurations $3 \mathrm{~s}^{-1} 3 \mathrm{p}^{-1}$ and $3 \mathrm{p}^{-3} 3 \mathrm{~d}$ are completely mixed in these states $[26,27]$. As a result, the Ar atomic Auger transition to the state at $70.65 \mathrm{eV}$ designated as $3 \mathrm{p}^{-3} 3 \mathrm{~d}{ }^{1} \mathrm{P}$ occurs with significant intensity [27]. This is also the case for the states at 57.56 and $69.94 \mathrm{eV}$. (Here, we have neglected the energy differences among the triplet states and taken the weighted average of their energies.) Although these states are assigned to $3 \mathrm{~s}^{-1} 3 \mathrm{p}^{-13} \mathrm{P}$ and $3 \mathrm{p}^{-3} 3 \mathrm{~d}^{3} \mathrm{P}$, respectively, these two configurations are also severely mixed. The Auger lines to the satellite state at $69.94 \mathrm{eV}$ also appear with some intensity. The intensity ratio of the Auger transitions to $3 \mathrm{p}^{-3} 3 \mathrm{~d}{ }^{1} \mathrm{P}$ and to $3 \mathrm{p}^{-3} 3 \mathrm{~d}^{3} \mathrm{P}$ is roughly $3: 1$ [27].

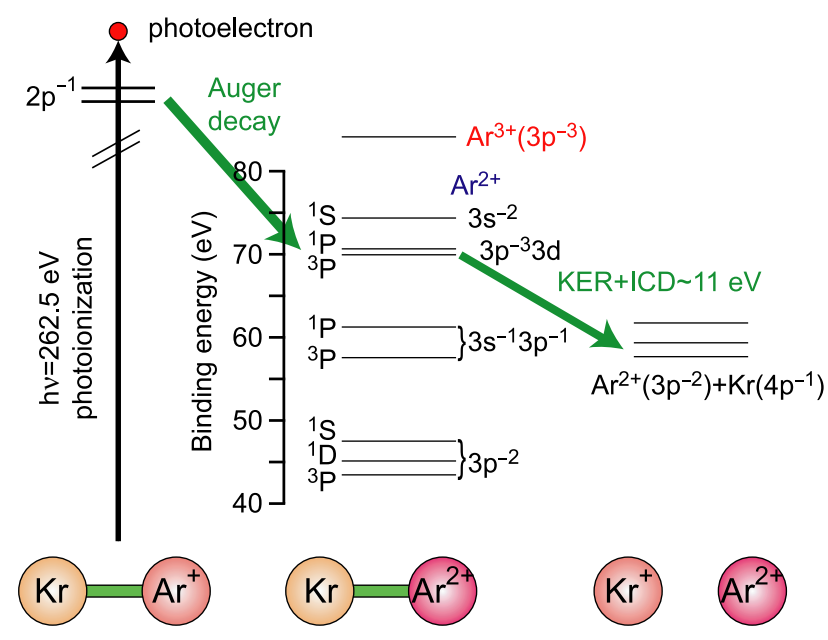

Figure 3. Schematic energy diagram for the states involved in the interatomic Coulombic decay. The triple ionization threshold of atomic $\mathrm{Ar}$ is also indicated in the figure.

The triple ionization threshold of atomic Ar $(84.12 \mathrm{eV})$ is higher than all of the Auger final states discussed above, and thus these states are not subject to autoionization in an isolated Ar atom. However, the triple ionization threshold for ArKr is significantly lower since the charge can be delocalized to the two sites. As a result, the states at 70.65 and 69.94 $\mathrm{eV}$, designated as $\mathrm{Ar}^{2+}\left(3 \mathrm{p}^{-3} 3 \mathrm{~d}\right){ }^{1} \mathrm{P}$ and ${ }^{3} \mathrm{P}$, respectively, are subject to ICD in the dimer (see figure 3). Although both the $\mathrm{Ar}^{2+}\left(3 \mathrm{p}^{-3} 3 \mathrm{~d}\right){ }^{1} \mathrm{P}$ and ${ }^{3} \mathrm{P}$ states are populated in atomic Auger decay only via the mixed configuration component $3 \mathrm{~s}^{-1} 3 \mathrm{p}^{-1}$, both the $3 \mathrm{p}^{-3} 3 \mathrm{~d}$ and the $3 \mathrm{~s}^{-1} 3 \mathrm{p}^{-1}$ configurations contribute to ICD: the $3 \mathrm{~d}$ electron (one of the $3 \mathrm{p}$ electrons) in the $3 \mathrm{p}^{-3} 3 \mathrm{~d}$ $\left(3 \mathrm{~s}^{-1} 3 \mathrm{p}^{-1}\right)$ configuration jumps into the $3 \mathrm{p}(3 \mathrm{~s})$ orbital in the Ar atom by emitting a virtual photon, while the $\mathrm{Kr}$ atom which absorbed the virtual photon emits a $4 p$ electron as an ICD electron. The possible final states are combinations of the doubly charged states of $\operatorname{Ar}^{2+}\left(3 \mathrm{p}^{-2}\right)$ and the singly charged states of $\mathrm{Kr}^{+}\left(4 \mathrm{p}^{-1}\right)$.

The possible ICD channels described above are listed in table 1. The two Auger final states $\mathrm{Ar}^{2+}\left(3 \mathrm{p}^{-3} 3 \mathrm{~d}\right){ }^{1} \mathrm{P}$ and ${ }^{3} \mathrm{P}$ are the initial states of the ICD and are listed with their energies (relative to the neutral ground state) at the top of the right-hand columns. The ICD final states corresponding to $\mathrm{Ar}^{2+}\left(3 \mathrm{p}^{-2}\right){ }^{1} \mathrm{~S},{ }^{1} \mathrm{D}$ and ${ }^{3} \mathrm{P}$ with $\mathrm{Kr}^{+}\left(4 \mathrm{p}^{-1}\right){ }^{2} \mathrm{P}_{1 / 2}$ and ${ }^{2} \mathrm{P}_{3 / 2}$ are listed with their energies in the first two columns. The possible combinations of the initial and final states of the ICD are labelled $\mathrm{A}-\mathrm{F}$ and $\mathrm{A}^{\prime}-\mathrm{F}^{\prime}$ in the table. The prime indicates a ${ }^{2} \mathrm{P}_{3 / 2}$ final state for $\mathrm{Kr}^{+}$. The listed energies correspond to the sum of the ICD electron energy and the KER, which can be estimated from the listed energies by $E\left(\operatorname{Ar}^{2+}\left(3 \mathrm{p}^{-3} 3 \mathrm{~d}\right)\right)-E\left(\operatorname{Ar}^{2+}\left(3 \mathrm{p}^{-2}\right)\right)-E\left(\operatorname{Kr}^{+}\left(4 \mathrm{p}^{-1}\right)\right)$.

The multiplicity of $\mathrm{Ar}^{2+}\left(3 \mathrm{p}^{-3} 3 \mathrm{~d}\right)$ in the ICD initial states is either singlet or triplet, and the multiplicity of $\mathrm{Ar}^{2+}\left(3 \mathrm{p}^{-2}\right)$ in the ICD final states is also either singlet or triplet. Let us assume that LSJ coupling is valid in Ar. Then, in the virtual photon exchange picture, the initial $\mathrm{Ar}^{2+}$ singlet (triplet) states decay to $\mathrm{Ar}^{2+}$ singlet (triplet) states by emitting a virtual photon, which is absorbed by $\mathrm{Kr}$ resulting in the emission of 
Table 1. Energy levels of the ICD initial states (Auger final states of $\mathrm{Ar}^{2+}$ relevant to the ICD) and the ICD final states of $\mathrm{Ar}{ }^{2+}$ and $\mathrm{Kr}^{+}$[26]. The labels of A-F correspond to those that appear in figure 2. The values after the labels A-F denote the sum of the ICD electron energy and the kinetic energy release (KER). The parentheses on the labels show that the spin is flipped in $\mathrm{Ar}^{2+}$ in the ICD.

\begin{tabular}{lllll}
\hline \multicolumn{2}{c}{ ICD final states } & & \multicolumn{3}{c}{ ICD initial states } \\
\cline { 1 - 1 } \cline { 5 - 5 } $\mathrm{Ar}^{2+}\left(3 \mathrm{p}^{-2}\right)$ & $\mathrm{Kr}^{+}\left(4 \mathrm{p}^{-1}\right)$ & & $\mathrm{Ar}^{2+}\left(3 \mathrm{p}^{-3} 3 \mathrm{~d}\right){ }^{1} \mathrm{P} 70.65$ & $\mathrm{Ar}^{2+}\left(3 \mathrm{p}^{-3} 3 \mathrm{~d}\right){ }^{3} \mathrm{P} 69.94$ \\
\hline${ }^{1} \mathrm{~S} 47.51$ & ${ }^{2} \mathrm{P}_{1 / 2} 14.67$ & $\mathrm{~A} 8.48$ & $(\mathrm{D} 7.76)$ \\
${ }^{1} \mathrm{D} 45.13$ & ${ }^{2} \mathrm{P}_{1 / 2} 14.67$ & $\mathrm{~B} 10.86$ & $(\mathrm{E} 10.15)$ \\
${ }^{3} \mathrm{P} 43.46$ & ${ }^{2} \mathrm{P}_{1 / 2} 14.67$ & & $(\mathrm{C} 12.53)$ & $\mathrm{F} 11.81$ \\
${ }^{1} \mathrm{~S} 47.51$ & ${ }^{2} \mathrm{P}_{3 / 2} 14.00$ & $\mathrm{~A}^{\prime} 9.14$ & $\left(\mathrm{D}^{\prime} 8.43\right)$ \\
${ }^{1} \mathrm{D} 45.13$ & ${ }^{2} \mathrm{P}_{3 / 2} 14.00$ & & $\mathrm{~B}^{\prime} 11.53$ & $\left(\mathrm{E}^{\prime} 10.81\right)$ \\
${ }^{3} \mathrm{P} 43.46$ & ${ }^{2} \mathrm{P}_{3 / 2} 14.00$ & & $\left(\mathrm{C}^{\prime} 13.20\right)$ & $\mathrm{F}^{\prime} 12.48$ \\
\hline
\end{tabular}

the ICD electron: the total spin in $\mathrm{Ar}^{2+}$ should be conserved in this way. One should however note that ICD can also take place via electron exchange, with a $\mathrm{Kr} 4 \mathrm{p}$ electron filling the $\mathrm{Ar}^{2+} 3 \mathrm{p}$ hole, and an $\mathrm{Ar}^{2+} 3 \mathrm{~d}$ electron being ejected as the ICD electron. In this case, the spin may appear to flip at the $\mathrm{Ar}^{2+}$ site. The labels in parentheses in table 1 indicate these spin-flip ICDs.

The ICD channels listed in table 1 are shown in figure 2(d) by labelled solid and dotted vertical lines. Lines B and B', corresponding to $E_{\text {sum }}=10.86$ and $11.53 \mathrm{eV}$, agree well with the maximum of the energy sum distribution. On the other hand, it is not possible to clearly identify any structure corresponding to line $\mathrm{C}^{\prime}$. Lines $\mathrm{B}$ and $\mathrm{B}^{\prime}$ correspond to spinconserved ICDs, whereas lines $\mathrm{C}$ and $\mathrm{C}^{\prime}$ correspond to spinflip ICDs. Thus, our observation clearly indicates significant suppression of the intensity of spin-flip ICDs in comparison with spin-conserved ICDs. The heights of the thick vertical lines correspond to the products of the relative populations of the ICD initial states as estimated from the Auger intensities (3:1 for ${ }^{1} \mathrm{P}:{ }^{3} \mathrm{P}$ ) and the statistical weights of the spin-conserved ICD final states. The spin-flip ICDs are indicated by thin lines, whose heights are scaled relative to the spin-conserved ICDs by the statistical weights of the final states. It is clear that the spin-conserved ICD channels A, $\mathrm{A}^{\prime}, \mathrm{F}$ and $\mathrm{F}^{\prime}$ also contribute, producing the broad energy-sum distribution of figure 2(d). Again we cannot clearly identify contributions from the spinflip ICDs to the intensity-sum distributions.

\section{Conclusion}

We have identified ICD processes from the Auger final states of the ArKr dimer by simultaneously determining the kinetic energy of the ICD electron and the KER between $\mathrm{Ar}^{2+}$ and $\mathrm{Kr}^{+}$using momentum-resolved electron-ion-ion coincidence spectroscopy. In the ArKr system the $\mathrm{Kr}$ atom can be regarded as the environment for the dicationic $\mathrm{Ar}^{2+}$ produced via $\mathrm{Ar}$ atomic Auger decay, and this environment opens ICD channels which are energetically forbidden for an isolated $\mathrm{Ar}$ atom. We find that spin-conserved ICDs, which can be viewed as energy transfer to the environment $(\mathrm{Kr})$ via virtual photon exchange, are significantly stronger than spin-flip ICDs. We note that ICDs following atomic Auger decay are very general decay channels which lead to the emission of low-energy electrons following inner-shell ionization. These processes are thus relevant to numerous physical, chemical and biological phenomena involving inner-shell vacancies in clusters and other forms of spatially extended atomic and molecular matter, including biomolecules in living cells.

\section{Acknowledgments}

The experiment was carried out with the approval of JASRI (2007A1602-NSb-np and 2007A1394-NSb-np). The authors are grateful to S Stoychev, A Kuleff, V Averbukh and LS Cederbaum for discussion on theoretical aspects of this study. The work was supported by grants in aid for scientific research provided by the Japan Society for Promotion of Science (JSPS), the budget for 'Promotion of X-ray Free Electron Laser Research' from the Japanese Ministry of Education, Culture, Sports, Science and Technology, the BMBF and DFG. XJL acknowledges financial support by JSPS. KK acknowledges financial support by DESY and the HGF Initiative and Networking Fund.

\section{References}

[1] Cederbaum L S, Zobeley J and Tarantelli F 1997 Phys. Rev. Lett. 794778

[2] Marburger S, Kugeler O, Hergenhahn U and Möller T 2003 Phys. Rev. Lett. 90203401

[3] Jahnke T et al 2004 Phys. Rev. Lett. 93163401

[4] Jahnke T, Weber Th, Osipov T, Landers A L, Jagutzki O, Schmidt L Ph H, Cocke C L, Prior M H, Schmidt-Böcking H and Dörner R 2004 J. Electron Spectrosc. Relat. Phenom. 141 229-38

[5] Ullrich J, Moshammer R, Dorn A, Dörner R, Schmidt L Ph H and Schmidt-Böcking H 2003 Rep. Prog. Phys. 66 1463-545

[6] Santra R, Zobeley J, Cederbaum L S and Moiseyev N 2000 Phys. Rev. Lett. 854490

[7] Averbukh V, Müller I B and Cederbaum L S 2004 Phys. Rev. Lett. 93263002

[8] Averbukh V and Cederbaum L S 2006 Phys. Rev. Lett. 96053401

[9] Kuleff A I and Cederbaum L S 2007 Phys. Rev. Lett. 98083201

[10] Öhrwall G et al 2004 Phys. Rev. Lett. 93173401

[11] Bradeanu I L, Flesch R, Meyer M, Jochims H-W and Rühl E 2005 Eur. Phys. J. D 36173

[12] Aoto T, Ito K, Hikosaka Y, Shigemasa E, Penent F and Lablanquie P 2006 Phys. Rev. Lett. 97243401

[13] Jahnke T et al 2007 Phys. Rev. Lett. 99153401

[14] Lundwall M et al 2007 J. Chem. Phys. 126214706 
[15] Santra R and Cederbaum L S 2003 Phys. Rev. Lett. 90153401 Santra R and Cederbaum L S 2005 Phys. Rev. Lett. 94 199901(E)

[16] Morishita Y et al 2006 Phys. Rev. Lett. 96243402

[17] Ohashi H, Ishiguro E, Tamenori Y, Kishimoto H, Tanaka M, Irie M, Tanaka T and Ishikawa T 2001 Nucl. Instrum. Methods A 467-468 529

[18] Tanaka T and Kitamura H 1996 J. Synchrotron Radiat. 347

[19] Ohashi H et al 2001 Nucl. Instrum. Methods A 467-468 533

[20] Fanis A De et al 2002 Phys. Rev. Lett. 89023006
[21] Saito N et al 2003 J. Phys. B: At. Mol. Opt. Phys. 36 L25

[22] Ueda K, Liu X-J, Prümper G, Fukuzawa H, Morishita Y and Saito N 2007 J. Electr. Spectrosc. Relat. Phenom. 155113

[23] Saito N, Liu X-J, Morishita Y, Suzuki I H and Ueda K 2007 J. Electron. Spectrosc. Relat. Phenom. 156-158 68

[24] Jagutzki O et al 2002 IEEE Trans. Nucl. Sci. 492477

[25] Ogilvie J F and Wang F Y H 1993 J. Mol. Struct. 291313

[26] http://physics.nist.gov/PhysRefData/ASD/levels_form.html

[27] Pulkkinen H, Aksela S, Sairanen O-P, Hiltunen A and Aksela H 1996 J. Phys. B: At. Mol. Opt. Phys. 293033 